\title{
Fantastic voyage: Catheter-based quantification of tracer distribution on a miniature scale
}

\author{
James T. Thackeray, $\mathrm{PhD}^{\mathrm{a}}$ \\ a Department of Nuclear Medicine, Hannover Medical School, Hannover, Germany
}

Received Sep 7, 2020; accepted Sep 8, 2020

doi: $10.1007 / \mathrm{s} 12350-020-02379-8$

\section{See related article, pp. 663-676}

The expansion of the nuclear cardiology armamentarium to encompass a wider range of tracers targeted to specific molecular processes offers opportunities for precise monitoring of disease progression and guidance of therapy. ${ }^{1}$ While some processes affect the global myocardium, many such as post-ischemic inflammation, infection, or fibroblast activation tend to be regional. ${ }^{2,3}$ Particularly subtle disease may also be below the detection limit of conventional imaging techniques. Moreover, localized pathology poses challenges to delivery of targeted cell, gene, or drug therapy, which may require precise targeting to evoke optimal benefit. As such, the limitations of resolution, regional heterogeneity, and mixed cellular substrates complicate interpretation of conventional radionuclide images, and raise the question whether other strategies could more precisely quantify regional tracer distribution.

In the current issue of the Journal of Nuclear Cardiology, Stendahl and colleagues describe a novel diagnostic device for minimally invasive tissue-level detection of tracer distribution in the myocardium, with a vision toward guided regional therapeutic intervention. ${ }^{4}$ The miniature plastic scintillator effectively discriminates $\beta+$ and $\beta-$ activity over $\gamma$-radiation, which could precisely pinpoint the spatial binding of a positron-emitting tracer. In a pig heart after ballooninduced myocardial infarction, heterogeneous uptake ${ }^{18} \mathrm{~F}$-fluorodeoxyglucose $\left({ }^{18} \mathrm{~F}-\mathrm{FDG}\right)$ was observed by ex vivo PET imaging, thought to reflect regional

\footnotetext{
Reprint requests: James T. Thackeray, PhD, Department of Nuclear Medicine, Hannover Medical School, Carl Neuberg Str 1, Hannover30625, Germany; Thackeray.James@mh-hannover.de J Nucl Cardiol 2022;29:677-9.

$1071-3581 / \$ 34.00$

Copyright (c) 2020 The Author(s)
}

inflammatory cell infiltration to the infarct and border zone territories. The regional disparity in signal was confirmed by spatial mapping using the $\beta$-detection catheter, providing a matrix distribution of activity content. Segment-to-segment activity established reasonable correlations between ex vivo PET, gamma well counting, and catheter-based measurements supporting the accuracy of the latter.

The strength of the manuscript lies in the ingenuity of the approach, which tackles the challenge of regional tracer uptake heterogeneity using a minimally invasive local measurement. While the analysis is limited to only two animals and selected areas in these infarct hearts, validation by conventional (albeit ex vivo) PET and well counting indicates the capacity of the $\beta$-detector catheter to define spatial disparities of tracer accumulation. However, it is disappointing that this potential is not explored further, e.g., by confirmation of cellular substrate by histopathology. Indeed, while typical investigation requires alignment of adjacent tissue sections for ex vivo autoradiography, histology, and immunohistochemistry, ${ }^{5,6}$ the $\beta$-detection catheter could better define the target regions of tracer enrichment to characterize the signal. A growing range of targeted inflammation radiotracers have displayed divergent uptake patterns in specific leukocyte subtypes in vitro with variable uptake in other cardiac cell subtypes including myocytes and fibroblasts. ${ }^{7,8}$ But in many cases the exact target cell type for these radiotracers remains equivocal. The development of radiotracers targeting reparative macrophage subtypes via mannose receptor, ${ }^{9}$ which are difficult to isolate within the mixed leukocytes in the damaged region, may be better identified by combining spatial catheter-based measurements with histology. It remains unclear how the spatial mapping of activity aligns to leukocytes, myocytes, and other support cells, which would provide greater support for the accuracy and applicability of this approach. Accordingly, further study should combine regionally focused $\beta$-detection with histopathology to determine the 
feasibility of image fusion and advance understanding of tracer substrates.

This limitation in regional characterization is compounded by the reliance on ${ }^{18} \mathrm{~F}$-FDG distribution for proof-of-concept. ${ }^{18} \mathrm{~F}-\mathrm{FDG}$ is notoriously promiscuous, which hampers the interpretation of the meaning of the signal. Indeed, numerous studies have demonstrated the inefficacy of typical suppression methods, especially in actively remodeling or acute infarct myocardium, generating a mixed substrate for the ${ }^{18}$ F-FDG signal including inflammatory leukocytes and metabolically compromised cardiomyocytes, ${ }^{3,10}$ though the regional heterogeneity observed in the porcine hearts and fasting protocols suggest preferential accumulation in infiltrating macrophages. Nonetheless, a more specific radiotracer could give greater clarity to the accuracy of the measurements.

A theoretical benefit of the $\beta$-detection catheter is the possibility to selectively distinguish $\beta+$ decay from $\gamma$ decay. Such detection might provide a more accurate localization of the signal by identifying the emitted particle rather than the annihilation event. With a rising prevalence of transition metal radiochemistry in cardiovascular molecular imaging, ${ }^{8}$ reconstruction accuracy is affected by wider positron range and partial volume effects. Regional mapping of activity concentration insulates the measurement from partial volume effects, particularly with thinning ventricle walls. In principle, the $\beta$-detection at the tissue level overcomes the spatial challenge of physics, accurately defining tracer distribution segment-by-segment.

Sensitivity poses a further challenge, wherein conventional PET imaging of inflammation after myocardial infarction has generally not attempted to discern between regionally robust or subtle inflammatory content. In conditions like myocardial infarction where the imaging target (i.e., leukocytes) is also elevated in the circulation, delineation of subtle tissue inflammation can be challenging, especially with transition metal isotopes. While the total inflammatory signal on PET imaging can predict functional outcome, ${ }^{5,11,12}$ subtle disease, and its role in the progression of heart failure is more difficult to assess. It is conceivable that $\beta$-detection catheter measurements could exhibit higher sensitivity for subtle disease, but this would require validation in vivo.

Indeed, it is problematic that the analysis is by necessity conducted entirely ex vivo. Clearly one benefit for the $\beta$-detection catheter is the possibility for minimally invasive measurement, providing a more accurate virtual biopsy on the miniature scale. It will be essential to demonstrate the equivalence of in vivo measurements, replete with cardiac motion to confirm the accuracy of catheter-based measurements in the real clinical situation. This will further allow characterization of $\beta$ detector sensitivity for activity in tissue sections against blood pool.

Nonetheless, the potential applications for such a device are broad, as many etiologies of cardiovascular disease exhibit regional heterogeneity. As intimated by Stendahl and colleagues, one potential application is localized inflammatory cell infiltration after acute myocardial infarction, where the severity and persistence of adverse inflammation can contribute to worse prognosis and remodeling. ${ }^{5,11}$ Clear delineation of regions with excessive inflammatory response may help to identify patients and regions that would most benefit from targeted therapy. Definitive characterization of the cellular substrate, as noted earlier, could also guide patients toward the most effective treatment based on the individual inflammatory pattern.

Ultimately, the greatest strength of the non-invasive or minimally invasive approach is the capability to combine regional diagnostics with regional therapy. Regional inflammation could be directly treated by antiinflammatory agents, gene therapy to promote endogenous repair, or immunomodulatory therapy to target adverse remodeling processes. ${ }^{13,14}$ Such approaches could overcome the hazards of systemic immunomodulation. Alternatively, prior studies have reported alignment of nuclear cardiac images to catheter-based measures of electrophysiology, where the site of inducibility of ventricular arrhythmia corresponded to lower retention of norepinephrine analogues. ${ }^{15,16}$ Integration of the $\beta$-detection capability with electrode measurements could provide further insights into this pathogenesis and provide a clearer map for ablation. The clinical relevance of the $\beta$-detection catheter is intimately linked to its potential theragnostic applications, which require dedicated investigation.

Miniaturization to explore pathogenesis remains the realm of science fiction, the capability to visualize distinct regional differences in tissue substrate within the injured myocardium on a miniatured scale is an initial step toward tissue-level quantification and substrate delineation. The potential combination of such tissuelevel imaging techniques with targeted delivery of cell-, gene-, or drug-based therapy bears clear potential for future modification of image-guided therapy. This catheter-based fantastic voyage provides a template for accurate study of regional tracer distribution, but many steps remain to bring such methodology to clinical practice.

\section{Disclosure}

Dr. Thackeray has no conflict of interest to declare. 


\section{Funding} DEAL

Open Access funding enabled and organized by Projekt

\section{Open Access}

This article is licensed under a Creative Commons Attribution 4.0 International License, which permits use, sharing, adaptation, distribution and reproduction in any medium or format, as long as you give appropriate credit to the original author(s) and the source, provide a link to the Creative Commons licence, and indicate if changes were made. The images or other third party material in this article are included in the article's Creative Commons licence, unless indicated otherwise in a credit line to the material. If material is not included in the article's Creative Commons licence and your intended use is not permitted by statutory regulation or exceeds the permitted use, you will need to obtain permission directly from the copyright holder. To view a copy of this licence, visit http://creativecommons.org/licenses/by/4.0/.

\section{References}

1. Hess A, Thackeray JT, Wollert KC, Bengel FM. Radionuclide image-guided repair of the heart. JACC Cardiovasc Imaging 2019. https://doi.org/10.1016/j.jcmg.2019.11.007.

2. Prabhu SD, Frangogiannis NG. The biological basis for cardiac repair after myocardial infarction: from inflammation to fibrosis. Circ Res 2016;119:91-112. https://doi.org/10.1161/CIRCRE SAHA.116.303577.

3. Glasenapp A, Derlin K, Wang Y, Bankstahl M, Meier M, Wollert $\mathrm{KC}$, et al. Multimodality imaging of inflammation and ventricular remodeling in pressure-overload heart failure. J Nucl Med 2020;61:590-6. https://doi.org/10.2967/jnumed.119.232488.

4. Stendahl JC, Liu Z, Boutagy NE, Nataneli E, Daghighian F, Sinusas AJ. Prototype device for endoventricualr beta-emitting radiotracer detection and molecularly-guided intervention. J Nucl Cardiol 2020. https://doi.org/10.1007/s12350-020-02317-8.

5. Thackeray JT, Hupe HC, Wang Y, Bankstahl JP, Berding G, Ross TL, et al. Myocardial inflammation predicts remodeling and neuroinflammation after myocardial infarction. J Am Coll Cardiol 2018;71:263-75. https://doi.org/10.1016/j.jacc.2017.11.024.

6. Taki J, Inaki A, Wakabayashi H, Matsunari I, Imanaka-Yoshida K, Ogawa K, et al. Postconditioning accelerates myocardial inflammatory resolution demonstrated by (14)C-methionine imaging and attenuates ventricular remodeling after ischemia and reperfusion. Circ J 2019;83:2520-6. https://doi.org/10.1253/circj.CJ-19-0462.
7. Borchert T, Beitar L, Langer LBN, Polyak A, Wester HJ, Ross TL, et al. Dissecting the target leukocyte subpopulations of clinically relevant inflammation radiopharmaceuticals. J Nucl Cardiol 2019. https://doi.org/10.1007/s12350-019-01929-z.

8. Heo GS, Sultan D, Liu Y. Current and novel radiopharmaceuticals for imaging cardiovascular inflammation. Q J Nucl Med Mol Imaging 2020;64:4-20. https://doi.org/10.23736/S1824-4785.20.0 3230-6.

9. Tahara N, Mukherjee J, de Haas HJ, Petrov AD, Tawakol A, Haider $\mathrm{N}$, et al. 2-Deoxy-2-[18F]fluoro-D-mannose positron emission tomography imaging in atherosclerosis. Nat Med 2014;20:215-9. https://doi.org/10.1038/nm.3437.

10. Thackeray JT, Bankstahl JP, Wang Y, Wollert KC, Bengel FM. Clinically relevant strategies for lowering cardiomyocyte glucose uptake for 18F-FDG imaging of myocardial inflammation in mice. Eur J Nucl Med Mol Imaging 2015;42:771-80. https://doi.org/10. 1007/s00259-014-2956-7.

11. Rischpler C, Dirschinger RJ, Nekolla SG, Kossmann H, Nicolosi $\mathrm{S}$, Hanus F, et al. Prospective evaluation of $18 \mathrm{~F}$-fluorodeoxyglucose uptake in postischemic myocardium by simultaneous positron emission tomography/magnetic resonance imaging as a prognostic marker of functional outcome. Circ Cardiovasc Imaging 2016;9:e004316. https://doi.org/10.1161/CIRCIMAGING.115. 004316.

12. Heo GS, Kopecky B, Sultan D, Ou M, Feng G, Bajpai G, et al. Molecular imaging visualizes recruitment of inflammatory monocytes and macrophages to the injured heart. Circ Res 2019;124:881-90. https://doi.org/10.1161/CIRCRESAHA.118. 314030 .

13. Aghajanian H, Kimura T, Rurik JG, Hancock AS, Leibowitz MS, $\mathrm{Li} \mathrm{L}$, et al. Targeting cardiac fibrosis with engineered $\mathrm{T}$ cells. Nature 2019;573:430-3. https://doi.org/10.1038/s41586-019-1546z.

14. Hess A, Derlin T, Koenig T, Diekmann J, Wittneben A, Wang Y, et al. Molecular imaging-guided repair after acute myocardial infarction by targeting the chemokine receptor CXCR4. Eur Heart J 2020. https://doi.org/10.1093/eurheartj/ehaa598.

15. Klein T, Abdulghani M, Smith M, Huang R, Asoglu R, Remo BF, et al. Three-dimensional 123I-meta-iodobenzylguanidine cardiac innervation maps to assess substrate and successful ablation sites for ventricular tachycardia: Feasibility study for a novel paradigm of innervation imaging. Circ Arrhythm Electrophysiol 2015;8:58391. https://doi.org/10.1161/CIRCEP.114.002105.

16. Sasano T, Abraham MR, Chang KC, Ashikaga H, Mills KJ, Holt $\mathrm{DP}$, et al. Abnormal sympathetic innervation of viable myocardium and the substrate of ventricular tachycardia after myocardial infarction. J Am Coll Cardiol 2008;51:2266-75. http s://doi.org/10.1016/j.jacc.2008.02.062.

Publisher's Note Springer Nature remains neutral with regard to jurisdictional claims in published maps and institutional affiliations. 